\title{
Evaluation in Arch Width Variations among Different Skeletal Patterns in District Solan Population
}

\author{
Isha Aggarwal' Sumit Chhatwalia' Sanjay Mittal' \\ ${ }^{1}$ Department of Orthodontics and Dentofacial Orthopedics, Bhojia \\ Dental College and Hospital, Himachal Pradesh, India
}

\author{
Mandeep K. Bhullar ${ }^{1}$ Divya Singla ${ }^{1}$
}

Address for correspondence Sumit Chhatwalia, MDS, Department of Orthodontics and Dentofacial Orthopedics, Bhojia Dental College and Hospital, Baddi, 176052, Himachal Pradesh, India (e-mail: torquing101186@gmail.com).

\author{
Abstract \\ Keywords \\ - normodivergent \\ - hypodivergent \\ - hyperdivergent \\ - interpremolar arch \\ widths \\ - intermolar arch widths
}

Introduction The facial growth pattern differs from individual to individual, and the variations in it are quite high. The assessment of relationship of dental arch dimensions with the facial growth pattern is essential for proper diagnosis and treatment planning. Aim The purpose of this study was to evaluate and compare the dental and alveolar arch widths in patients with varying facial growth patterns in Distt. Solan population. Materials and Methods Pretreatment lateral cephalograms and dental study models of 45 patients with age group between 16 and 30 years were included in the study. Patients were divided into three groups: group I (normodivergent), group II (hypodivergent), and group III (hyperdivergent) on the basis of y-axis, Jarabak ratio, and SN-MP (Sella-Nasion-mandibular plane) angle. Interpremolar and intermolar dental and alveolar arch widths were measured and compared for all the three groups.

Results The results showed that the dental and alveolar arch widths were increased in hypodivergent patients and decreased in hyperdivergent patients, which was not statistically significant.

Conclusion It was concluded that the dental and alveolar arch dimensions increased as the facial pattern became horizontal.

\section{Introduction}

Improving the aesthetics of the face is one of the main reasons for a patient seeking orthodontic treatment. This can only be achieved by thorough diagnosis, which involves intra- and extraoral measurements of the face and dental arches. ${ }^{1}$ The dental arches change due to intervention in treatment as well as with growth and development. The proper identification of a patient's arch form is an important characteristic of attaining a stable, functional, and aesthetic result of orthodontic treatment, and failing in preserving the arch form might increase the chances of relapse. ${ }^{2}$ Arch form is the position and relationship of teeth to each other in all three dimensions. ${ }^{3}$ Several studies have shown the relationship of arch forms and dimensions, particularly the arch widths with other dentoskeletal features. ${ }^{4}$

Dental arch widths and facial forms are key factors for ascertaining success and of orthodontic treatment. It is credited that a relationship is there between arch widths and facial forms. ${ }^{2}$ Facial morphology has been believed to be

\section{received}

December 4, 2018

accepted after revision

December 22, 2018

published online

January 29, 2019 the outcome of each person's genotypic and its phenotypic expression. Three basic types of facial morphology exist: normodivergent, hypodivergent, and hyperdivergent. Hypodivergent patients (brachyfacial) are characterized by wider arch dimensions, and hyperdivergent patients (dolichofacial) are characterized by narrower arch dimensions according to Ricketts et al (1982). ${ }^{5}$ The two paramounts of vertical facial dysplasia were also explained as hypodivergent and hyperdivergent growth pattern by Schudy $(1964)^{6}$ or short- and long-face syndrome by Opdebeeck and Bell (1978). ${ }^{7}$ The maxillary and mandibular dental arches can be considered as kind of ribbons, adapted to altering jaw relationships to maintain normal association between the arches for esthetic and function. ${ }^{8}$

Schudy $(1964)^{6}$ recommended use of anterior cranial base (Sella-Nasion [SN] plane) as reference line to establish the inclination of the mandibular plane (MP). A patient with a high SN-MP angle (steep MP) has a tendency of longer face, and one with a smaller SN-MP angle (flat MP) tends to have a shorter face. Howes (1957) ${ }^{9}$ reported that increased MP angle 
individuals predominantly had teeth with increased size, narrower and shorter arches than decreased MP individuals when observed from the buccal cusp tips of the maxillary first bicuspids. Isaacson et al (1971) ${ }^{10}$ found that individuals with long faces dispensed with a reduced maxillary intermolar width. Nasby et al (1972) $)^{11}$ stated that the mandibular intermolar width were increased in patients with reduced SN-MP angles when differentiated with those with high SN-MP angles. Eröz et al $(2000)^{12}$ stated that males had significantly greater intermolar widths juxtaposed to females.

Dental arches manifesting ideal intercuspation of the teeth and balanced function are elemental for identifying occlusion and sustaining its stability. Functional changes cause physiologic disparity in the muscle constraint deployed on dental arches, often developing discrepancies such as deficient maxilla. The activity of muscle of mastication has effects on occlusion, dental arch form, and shape of the mandible. ${ }^{13}$ The correlation between characteristics of dental arch and vertical pattern of face can assist the clinician to individualize the treatment, thereby enhancing the orthodontic therapy response. The practitioner should know the attributes of normality according to age, sex, ethnicity, and facial type. Although little research was done regarding vertical facial pattern and arch dimensions. ${ }^{14}$ Thus this study was carried out to assess the alliance between dental and alveolar arch dimensions and vertical facial pattern.

\section{Aim}

The purpose was to evaluate dental and alveolar arch widths in patients with varying growth patterns. The objectives were as follows:

- To measure and collate the dental arch dimensions (interpremolar and intermolar widths) in patients with varying growth patterns.

- To measure and collate the alveolar arch dimensions (interpremolar and intermolar widths) in patients with varying growth patterns.

\section{Materials and Methods}

This study was organized in the Department of Orthodontics and Dentofacial Orthopaedics at Bhojia Dental College, Baddi, Himachal Pradesh. The study was performed using pretreatment lateral cephalograms and dental study models of 45 patients who were reported to the department for fixed orthodontic treatment. Patients' age ranged between 16 and 30 years. The following inclusion and exclusion criteria were used for selection.

\section{Inclusion Criteria}

- All secondary dentition should be present irrespective of third molars.

- Patients with any type of skeletal or dental malocclusion.

\section{Exclusion Criteria}

- Patients with anterior and posterior cross bites.

- Patients with excessive crowding (>9 $\mathrm{mm}$ ) and spacing (>9 mm).
- Patients with any craniofacial anomalies or any syndrome.

- History of trauma to dentofacial region.

- Cuspal wear due to any deleterious habit or any other reason.

- Any gross carious lesions or any excessive restorations and prosthetics.

Patients were divided into groups of three with 15 each on the basis of $\mathrm{y}$-axis, Jarabak ratio (J ratio), and SN-MP angle.

All the reference points were marked with $4 \mathrm{H}$ pencil on the study models (-Table $\mathbf{1}$ ). All measurements were done using a digital vernier caliper by the same operator ( - Fig. 1 ).

The maxillary and mandibular dimensions measured were as follows:

- Interpremolar dental arch width (IPW) (-Figs. 2, 3): Interpremolar width is defined as the distance measured between the buccal cusp tips of right and left first premolars. ${ }^{15}$

- Intermolar dental arch width (IMW) (-Figs. 2, 3): Intermolar width is defined as the distance measured from the mesiobuccal cusp tips of right to left maxillary or mandibular first molars. ${ }^{15}$

- Premolar basal arch width (PMBAW) (- Figs. 2, 3): Premolar basal arch width is defined as the distance measured between the two points at the mucogingival junctions above the interdental contact points of the maxillary first and second premolars and below the interdental contact points of the mandibular first and second premolars, respectively. ${ }^{15}$

- Molar basal arch width (MBAW) (-Figs. 2, 3): Molar basal arch width is defined as the distance between the two points at mucogingival junction above the mesiobuccal cusp tips of the maxillary first molars and below the mesiobuccal cusp tips of the mandibular first molars, respectively. ${ }^{15}$

Table 1 Grouping of patients

\begin{tabular}{|l|l|l|l|}
\hline Groups & Y-axis $\left(^{\circ}\right)$ & J ratio $\left(^{\circ}\right)$ & $\begin{array}{l}\text { SN-MP angle } \\
\left(^{\circ}\right)\end{array}$ \\
\hline $\begin{array}{l}\text { Group I }(n=15) \\
\text { Hyperdivergent }\end{array}$ & $>61.2$ & $>65$ & $>26$ \\
\hline $\begin{array}{l}\text { Group II }(n=15) \\
\text { Normodivergent }\end{array}$ & $59.4 \pm 2$ & $62-65 \%$ & $24 \pm 2$ \\
\hline $\begin{array}{l}\text { Group III } \\
(n=15) \\
\text { Hypodivergent }\end{array}$ & $<57.4$ & $<62$ & $<22$ \\
\hline
\end{tabular}

Abbreviations: J ratio, Jarabak ratio; SN-MP, Sella-Nasion-mandibular plane.

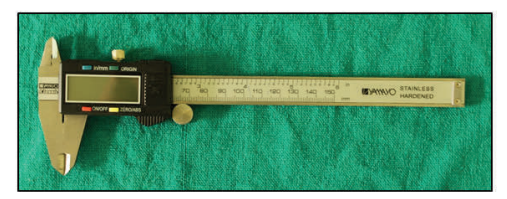

Fig. 1 Digital vernier caliper (Yamayo Digimatic caliper). IMW, intermolar dental arch width; IPW, interpremolar dental arch width; MBAW, molar basal arch width; PMBAW, premolar basal arch width. 


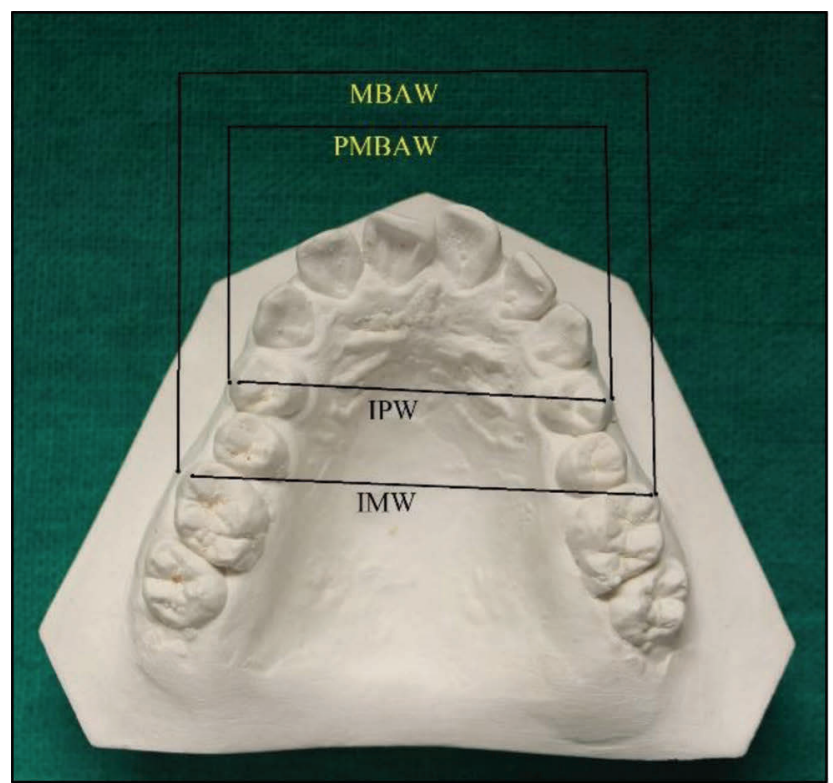

Fig. 2 Interpremolar and intermolar dental and alveolar arch width (maxilla).

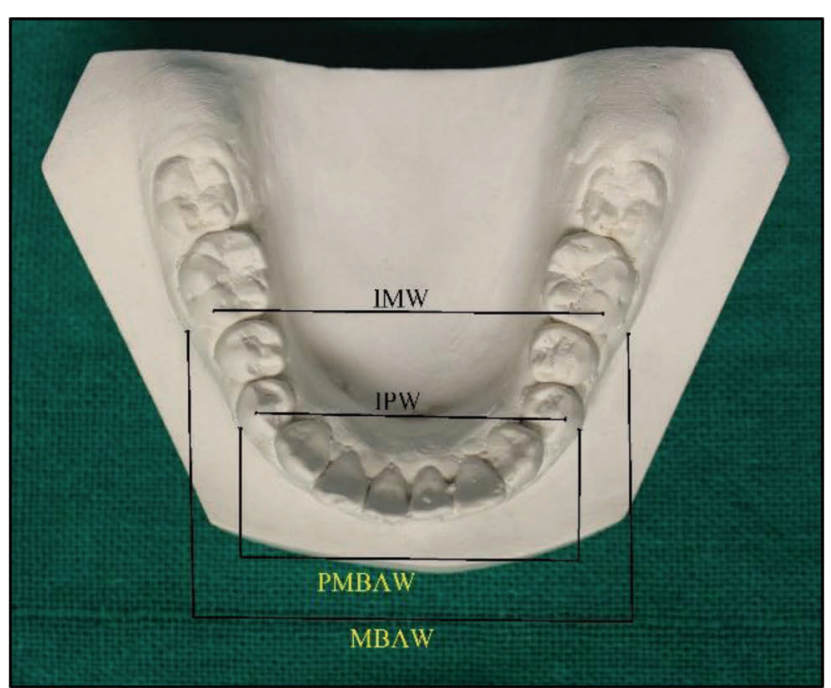

Fig. 3 Interpremolar and intermolar dental and alveolar arch width (mandibular). IMW, intermolar dental arch width; IPW, interpremolar dental arch width; MBAW, molar basal arch width; PMBAW, premolar basal arch width.

\section{Statistical Analysis}

The data so obtained were applied for the statistical analysis using statistical package program SPSS software version 10.2 (IBM; New York, United States). Descriptive statistics, including the mean and standard deviation (SD) values, were calculated for all the parameters in each group. Analysis of variance (ANOVA) test was carried out to show the intergroup comparisons. Post hoc tests were done for multiple comparisons.

\section{Results}

The study was conducted on dental casts' model of 45 patients who were separated into three groups, that is, group I (hyperdivergent), group II (normodivergent), and group III (hypodivergent), with 15 in each group on the basis of SN-MP angle, J ratio, and y-axis. The two measurements from each maxilla and mandible were carried out on the casts' models using a vernier calliper.

The descriptive statistics showed that the mean interpremolar dental arch width was found to be highest in normodivergent group $(43.03 \mathrm{~mm})$ than in hypodivergent group (42.06 mm) and lowest in hyperdivergent group (41.96 mm) for maxillary study models as depicted in - Table 2. The descriptive statistics showed that mean intermolar dental arch width was found to be highest in normodivergent group ( $52.86 \mathrm{~mm}$ ) than in hypodivergent group $(52.70 \mathrm{~mm})$ and lowest in hyperdivergent group (52.46 mm) for maxillary study models, as depicted in - Table 2 .

The descriptive statistics showed that mean interpremolar alveolar arch width was found to be highest in normodivergent group (51.13 $\mathrm{mm}$ ) than the hypodivergent group (49.16 $\mathrm{mm}$ ) and lowest in hyperdivergent group (48.46 $\mathrm{mm}$ ) for maxillary study models as depicted in - Table 2. The descriptive statistics showed that mean intermolar alveolar arch width was also found to be highest in normodivergent group (59.13 $\mathrm{mm}$ ) than the hypodivergent group (58.86 mm) and lowest in hyperdivergent group $(58.06 \mathrm{~mm})$ for maxillary study models as depicted in - Table 2 .

The descriptive statistics showed that the mean interpremolar dental arch width was found to be highest in normodivergent group (35.93 $\mathrm{mm}$ ) than in hypodivergent group (35 mm)and lowest in hyperdivergentgroup $(34.50 \mathrm{~mm})$

Table 2 Descriptive statistics for maxillary arch

\begin{tabular}{|l|l|l|l|l|l|l|l|}
\hline Parameters & \multicolumn{4}{|l|}{ Hyperdivergent $(\boldsymbol{n}=15)$} & Normodivergent $(\boldsymbol{n}=15)$ & \multicolumn{4}{l|}{ Hypodivergent $(\boldsymbol{n}=15)$} & ANOVA $\boldsymbol{p}$-Value \\
\hline & Mean SD & $\begin{array}{l}\text { Standard } \\
\text { error }\end{array}$ & Mean SD & $\begin{array}{l}\text { Standard } \\
\text { error }\end{array}$ & Mean SD & $\begin{array}{l}\text { Standard } \\
\text { error }\end{array}$ & \\
\hline IPW Mx & $41.967 \pm 3.02$ & 0.782 & $43.033 \pm 3.58$ & 0.925 & $41.96 \pm 3.90$ & 1.007 & 0.660 \\
\hline IMW Mx & $52.46 \pm 3.44$ & 0.888 & $52.86 \pm 3.73$ & 0.965 & $52.70 \pm 4.43$ & 1.145 & 0.961 \\
\hline PMBAW Mx & $48.46 \pm 3.58$ & 0.925 & $51.13 \pm 3.61$ & 0.934 & $49.16 \pm 3.83$ & 0.990 & 0.133 \\
\hline MBAW Mx & $58.06 \pm 4.61$ & 1.192 & $59.13 \pm 2.94$ & 0.761 & $58.86 \pm 3.96$ & 1.022 & 0.740 \\
\hline
\end{tabular}

Abbreviations: ANOVA, analysis of variance; IMW, intermolar dental arch width; IPW, interpremolar dental arch width; MBAW, molar basal arch width; PMBAW, premolar basal arch width; SD, standard deviation. 
for mandibular study models as depicted in - Table $\mathbf{3}$. The descriptive statistics showed that mean intermolar dental arch width was found to be highest in normodivergent group $(46.06 \mathrm{~mm})$ than in hypodivergent group $(45.76 \mathrm{~mm})$ and lowest in hyperdivergent group $(45.70 \mathrm{~mm})$ for mandibular study models as depicted in - Table 3 .

The descriptive statistics showed that mean interpremolar alveolar arch width was found to be highest in normodivergent group $(44.20 \mathrm{~mm})$ than the hypodivergent group $(42.80 \mathrm{~mm})$ and lowest in hyperdivergent group $(42.06 \mathrm{~mm})$ for mandibular study models as depicted in - Table 3. The descriptive statistics showed that mean intermolar alveolar arch width was also found to be highest in normodivergent group (55.43 $\mathrm{mm}$ ) than the hypodivergent group $(54.30 \mathrm{~mm}$ ) and lowest in hyperdivergent group $(54.26 \mathrm{~mm})$ for mandibular study models as depicted in - Table 3 .

ANOVA statistics was done to compare the arch dimensions among the three groups. The results showed that the interpremolar and intermolar dental and alveolar arch widths were not statistically significant $(p>0.05)$ when intergroup comparison was made, as depicted in - Tables 2 and 3. For the multiple comparisons, post hoc test was done by Tukey HSD method. The results for multiple comparisons showed that no statistical difference was found to be significant $(p>0.05)$ when the interpremolar and intermolar dental and alveolar arch widths of the three groups were compared, as depicted in $\mathbf{- T a b l e} 4$.

\section{Discussion}

Vertical facial pattern is an important element of orthodontic assessment. It is an essential criterion for each orthodontist to understand the association between vertical facial height and dental arch width for proper diagnosis and treatment planning. ${ }^{16}$

The facial growth pattern differs from individual to individual, and the variations in it are quite high. The assessment of relationship of dental arch dimensions with the vertical facial pattern is essential to understand the differential in size and shape of dental arches. It has been suggested that an individual with a greater SN-MP angle have a tendency of longer face and narrower arch dimensions and in individual with a reduced SN-MP angle predominantly has a shorter face and wider arch dimensions (Ricketts et al [1981],, Enlow and Hans $[1996]^{17}$ ). This study was conducted to interpret and collate the interpremolar and intermolar dental and alveolar arch dimensions in patients with varying growth patterns. Forty-five patients were selected for this study. These were divided into three groups with 15 in each: group I (hyperdivergent), group II (normodivergent), and group III (hypodivergent) on the basis of $\mathrm{J}$ ratio, $\mathrm{y}$-axis, and SN-MP angle. J ratio was used because it is a reliable measurement, constructed from anatomic landmarks (Bishara and Jacobsen $\left.[1985]^{18}\right)$, and the chance of human error is also minimized by using a ratio instead of linear parameter.

Table 3 Descriptive statistics for mandibular arch

\begin{tabular}{|l|l|l|l|l|l|l|l|}
\hline Parameters & Hyperdivergent $(\boldsymbol{n}=15)$ & Normodivergent $(\boldsymbol{n}=15)$ & \multicolumn{4}{l|}{ Hypodivergent $(\boldsymbol{n}=15)$} & ANOVA $p$-Value \\
\hline & Mean SD & Standard error & Mean SD & $\begin{array}{l}\text { Standard } \\
\text { error }\end{array}$ & Mean SD & $\begin{array}{l}\text { Standard } \\
\text { error }\end{array}$ & \\
\hline IPW Md & $34.50 \pm 3.09$ & 0.798 & $35.93 \pm 3.98$ & 1.028 & $35.00 \pm 3.31$ & 0.856 & 0.525 \\
\hline IMW Md & $45.70 \pm 4.13$ & 1.068 & $46.06 \pm 3.57$ & 0.921 & $45.76 \pm 3.45$ & 0.891 & 0.960 \\
\hline PMBAW Md & $42.06 \pm 3.00$ & 0.775 & $44.20 \pm 2.71$ & 0.701 & $42.80 \pm 2.85$ & 0.738 & 0.129 \\
\hline MBAW Md & $54.26 \pm 3.12$ & 0.806 & $55.43 \pm 2.63$ & 0.681 & $54.30 \pm 3.33$ & 0.860 & 0.496 \\
\hline
\end{tabular}

Abbreviations: ANOVA, analysis of variance; IMW, intermolar dental arch width; IPW, interpremolar dental arch width; MBAW, molar basal arch width; PMBAW, premolar basal arch width; SD, standard deviation.

Table 4 Multiple comparisons post hoc

\begin{tabular}{|l|l|l|l|l|l|l|}
\hline & \multicolumn{2}{l|}{ Normo vs. hypo } & \multicolumn{2}{l|}{ Hyper vs. hypo } & \multicolumn{2}{l|}{ Normo vs. hyper } \\
\hline & Mean diff. & $p$-Value & Mean diff. & $p$-Value & Mean diff. & $p$-Value \\
\hline IPW Mx & 1.066 & 0.687 & 1.000 & 0.997 & 0.966 & 0.734 \\
\hline IMW Mx & 0.166 & 0.992 & 0.233 & 0.985 & 0.400 & 0.957 \\
\hline PMBAW Mx & 2.666 & 0.129 & 0.700 & 0.862 & 1.966 & 0.319 \\
\hline MBAW Mx & 1.066 & 0.736 & 0.800 & 0.841 & 0.266 & 0.981 \\
\hline IPW Md & 1.433 & 0.503 & 0.500 & 0.919 & 0.933 & 0.745 \\
\hline IMW Md & 0.300 & 0.974 & 0.066 & 0.999 & 0.366 & 0.961 \\
\hline PMBAW Md & 1.400 & 0.382 & 0.733 & 0.115 & 2.133 & 0.764 \\
\hline MBAW Md & 1.166 & 0.550 & 0.033 & 1.000 & 1.133 & 0.569 \\
\hline
\end{tabular}

Abbreviations: IMW, intermolar dental arch width; IPW, interpremolar dental arch width; MBAW, molar basal arch width; PMBAW, premolar basal arch width. 
Four arch width computations were included for the maxillary and mandibular study models: interpremolar dental arch width, intermolar dental arch width, interpremolar alveolar arch width, and intermolar alveolar arch width.

The results showed that in maxillary and mandibular arches there is an inverse correlation among the morphology of face and dental arch widths (-Tables 2,3). The mean interpremolar, intermolar dental and interpremolar, intermolar alveolar arch width in both maxillary and mandibular arches were found to be highest in hypodivergent patients and least in hyperdivergent patients, which was not found to be statistically significant. This is in conformance with the researches done by Uysal et al (2005), ${ }^{15}$ Khera et al (2012), ${ }^{8}$ Ribeiro et al (2012), ${ }^{19}$ Grippaudo et al (2013), ${ }^{20}$ Bhutta et al (2013), ${ }^{1}$ Shahroudi and Etezadi (2013), ${ }^{21}$ Prasad et al (2013), ${ }^{22}$ Bălan et al (2014),,3 Nayer et al (2015), ${ }^{24}$ Traldi et al (2015), ${ }^{25}$ Gupta and Makhija (2016),, ${ }^{26}$ Perez et al (2016), ${ }^{27}$ Aditi et al (2017), ${ }^{28}$ and Nagrajmurthy (2017). ${ }^{29}$

However, the data from this study presented an antithetical fashion between SN-MP angle and dental arch widths, the relationship was less strong, which showed that the SN-MP angle might not be only one of the contributing factors. ${ }^{21}$

ANOVA ( - Table 2) shows that arch widths decreased with an increase in SN-MP angle. Isaacson et al (1979) ${ }^{10}$ reported that steep MP individuals generally had narrower maxillary first intermolar width than flat MP individuals. They suggested that the backward rotation of mandible in high SN-MP cases cause an increase in facial height that tends to lengthen the musculature, resulting in narrowing of the dental arch. Conversely, the low SN-MP cases cause a decrease in facial height that tends to permit maxillary teeth to move toward buccoversion, resulting in widening of the dental arch. Nasby et al (1972) ${ }^{11}$ also reported that backward rotating mandibles (hyperdivergent pattern) were associated with narrower intermolar widths. Various authors reported that patients with greater vertical dimensions have tendency of posterior teeth to be more buccally inclined, whereas those with reduced vertical dimensions have tendency of posterior teeth toward more lingual inclination, as per studies by Isaacson et al (1971), ${ }^{10}$ Schudy et al (1971), ${ }^{30}$ Schendel et al (1976), ${ }^{31}$ and Janson et al (2004). ${ }^{32}$

When multiple comparisons (-Table 4) were made between all the three groups, it was found out that transverse dimensions increase as the facial pattern becomes more horizontal (hypodivergent), which was not statistically significant in this study. This finding can be attributed to the muscle pattern of the individual, which has been regarded as the feasible link in their close association between transverse dimensions and vertical facial morphology.

Various researches have demonstrated the effect of masticatory muscles on craniofacial growth. ${ }^{33-35}$ The general concord is that patients with strong or thick mandibular elevator muscles have a tendency of manifesting wider transverse head dimensions (Ringqvist [1973], ${ }^{36}$ Ingervall and Helkimo [1978], ${ }^{37}$ Weijs and Hillen [1984], ${ }^{34}$ Hannam and Wood [1989],,38 Kiliaridis and Kälebo [1991], ${ }^{39}$ van Spronsen et al [1991], ${ }^{40}$ Bakke et al [1992],, Kiliaridis [1995] ${ }^{42}$ ). Strong musculature of mastication is habitually associated with a brachyfacial pattern (short face). This muscular hyper function causes a greater mechanical loading of the jaws. This may cause an initiation of growth at sutures and bone apposition, which consequently causes enhanced transverse growth of the dental arches and bone bases. ${ }^{43}$ On the contrary, van Spronsen et $\mathrm{al}^{44}$ reported that individuals with long face have significantly smaller masseter and medial pterygoid muscles than normal individuals (Satirğlu et al, ${ }^{35}$ Weijs and Hillen, ${ }^{45}$ Kiliardis and Kälebo, ${ }^{39}$ and Benington et $\mathrm{al}^{46}$ ).

Morphologic features related to masticatory functions, and facial types have been correlated with thickness of cortical bone of the mandible and buccolingual inclination of the first and second molars. The activity of muscles of mastication has effects on occlusion, form of dental arches, and shape of mandible. Mandibular molars erupt lingually and then buccally incline by the tongue pressure and masticatory function setting in a position of equilibrium between lingual and buccal pressures.

When the lingual volume of patients with long face is same as that of short-face ones, their molars sustain a great pressure despite the narrowed arch and verticalization occur as a consequence. The thickness of buccal cortical bone is greater in short-face patients than in long-face ones, and during the masticatory function, teeth are reinforced by this enormous bone structure, paramounting to a lingual inclination more than that in patients with mean and vertical facial types. ${ }^{33}$

The prediction of the interarch width helps us in situations such as cross bites, ectopically positioned teeth, transpositions, scissors bite, impacted teeth, missing teeth, etc., where we cannot determine exact interarch widths and fabricate customized arch wires for the patients. ${ }^{22}$ Thus to acquire a improved arch form, it is advisable to attain more posttreatment solidity; hence, the purpose of most studies on dental arch forms is to reveal whether the preformed arch wires can be ligated in all patients. ${ }^{20}$ The limitations of this research must be recognized because variation among individuals faced and dental arch dimensions are certainly a multifactorial phenomenon. ${ }^{47}$

\section{Conclusion}

The following conclusions were drawn from this study:

- The maxillary and mandibular interpremolar dental and alveolar width was highest in hypodivergent individuals and least in hyperdivergent individuals.

- The maxillary and mandibular intermolar dental and alveolar width was highest in hypodivergent individuals and least in hyperdivergent individuals.

- The transverse dimensions increase as the facial pattern becomes more horizontal.

\section{Conflict of Interest}

None declared.

\section{References}

1 Bhutta N, Israr J, Ijaz A. Comparison of dental and alveolar arch widths in class I and class II division 1 malocclusion. Pak Oral Dent J 2013;33(2):289-294

2 Bayome M, Sameshima GT, Kim Y, Nojima K, Baek SH, Kook YA. Comparison of arch forms between Egyptian and North American white populations. Am J Orthod Dentofacial Orthop 2011;139(3):e245-e252 
3 Lee RT. Arch width and form: a review. Am J Orthod Dentofacial Orthop 1999;115(3):305-313

4 Fleming PS, Dibiase AT, Lee RT. Arch form and dimensional changes in orthodontics. Prog Orthod 2008;9(2):66-73

5 Ricketts RM, Roth RH, Chaconas SJ, Schulhof RJ, Engel GA. Orthodontic Diagnosis and Planning. Denver, CO: Rocky Mountain Data Systems; 1982

6 Schudy FF. Vertical growth versus anteroposterior growth as related to function and treatment. Angle Orthod 1964;34(2):75-93

7 Opdebeeck H, Bell WH. The short face syndrome. Am J Orthod 1978;73(5):499-511

8 Khera AK, Singh GK, Sharma VP, Singh A. Relationship between dental arch dimensions and vertical facial morphology in class I subjects. J Indian Orthod Soc 2012;46(6):316-324

9 Howes AE. Arch width in the premolar region-still the major problem in orthodontics. Am J Orthod Dentofacial Orthopedics 1957;43(1):5-31

10 Isaacson JR, Isaacson RJ, Speidel TM, Worms FW. Extreme variation in vertical facial growth and associated variation in skeletal and dental relations. Angle Orthod 1971;41(3):219-229

11 Nasby JA, Isaacson RJ, Worms FW, Speidel TM. Orthodontic extractions and the facial skeletal pattern. Angle Orthod 1972;42(2):116-122

12 Eröz UB, Ceylan I, Aydemir S. An investigation of mandibular morphology in subjects with different vertical facial growth patterns. Aust Orthod J 2000;16(1):16-22

13 Gallão S, Faltin K Jr, Santos-Pinto L, Santos-Pinto A, Martins LP. Facial type measurements influence on transverse dimensions of normal occlusion arches. J Health Sci Inst. 2013;31(3):20-23

14 Ursi WJ, Trotman CA, McNamara JA Jr, Behrents RG. Sexual dimorphism in normal craniofacial growth. Angle Orthod 1993;63(1):47-56

15 Uysal T, Memili B, Usumez S, Sari Z. Dental and alveolar arch widths in normal occlusion, class II division 1 and class II division 2. Angle Orthod 2005;75(6):941-947

16 Anwar N, Fida M. Clinical applicability of variations in arch dimensions and arch forms among various vertical facial patterns. J Coll Physicians Surg Pak 2011;21(11):685-690

17 Enlow DH, Hans MG. Essentials of Facial Growth. Philadelphia, PA: WB Saunders; 1996

18 Bishara SE, Jakobsen JR. Longitudinal changes in three normal facial types. Am J Orthod 1985;88(6):466-502

19 Ribeiro JS, Ambrosio AR, Santos-Pinto A, Shimizu IA, Shimizu $\mathrm{RH}$. Evaluation of transverse changes in the dental arches according to growth pattern: a longitudinal study. Dental Press J Orthod 2012;17(1):66-73

20 Grippaudo C, Oliva B, Greco AL, Sferra S, Deli R. Relationship between vertical facial patterns and dental arch form in class II malocclusion. Prog Orthod 2013;14:43

21 Shahroudi AS, Etezadi T. Correlation between dental arch width and sagittal dento-skeletal morphology in untreated adults. J Dent (Tehran) 2013;10(6):522-531

22 Prasad M, Kannampallil ST, Talapaneni AK, George SA, Shetty SK. Evaluation of arch width variations among different skeletal patterns in South Indian population. J Nat Sci Biol Med 2013;4(1):94-102

23 Bălan RA, Popa G, Biță R, Fabricky M, Jivănescu A, Bratu DC. Alveolar and dental arch morphology in Angle class II division 2 malocclusion: a comparative study. Rom J Morphol Embryol 2014;55(3, Suppl):1093-1097

24 Nayar S, Aruna S, Manzoor W. Correlation between arch form and facial for a cross sectional study. J Pharm Bioallied Sci 2015;7(Suppl 1):S85-S86

25 Traldi A, Valdrighi HC, de Souza LZ, Vedovello SAS. Evaluation of facial morphology and sagittal relationship between dental arches in primary and mixed dentition. Dental Press J Orthod 2015;20(4):63-67
26 Gupta M, Makhija PG. Quantitative evaluation of masseter muscle volume in different growth patterns and its correlation with facial morphology: Cone beam computed tomography study. J Indian Orthod Soc 2016;50:106-110

27 Perez TLB, Santna YJK, Ruiz GEC, Carvajal AMS. Correlation of facial profile and dental arches in a population of Yucatan. Rev Mex Ortodon 2016;4(2):e81-e84

28 Aditi M, Anmol M, Vikram Pal A. A comparative study to access cephalometric and arch width characteristics of class II division 1 and division 2 malocclusions among Indian and Turkish population. J Dent Sci 2017;2(3):000133

29 Nagrajmurthy Thimmaiah VT. Comparative study of MDCT with plaster cast model of dentoalveolar arch morphology. Int J Anat Rad Surg 2017;6(2):RO17-RO20

30 Schudy FF. Cant of the occlusal plane and axial inclinations of the teeth. Angle Orthod 1971;41:219-229

31 Schendel Stephen A. The long face syndrome: vertical maxillary excess. Am J Orthod Dentofac Orthop 70(4):398-408

32 Janson G, Bombonatti R, Cruz KS, Hassunuma CY, Del Santo $\mathrm{M}$ Jr. Buccolingual inclinations of posterior teeth in subjects with different facial patterns. Am J Orthod Dentofacial Orthop 2004;125(3):316-322

33 Tsunori M, Mashita M, Kasai K. Relationship between facial types and tooth and bone characteristics of the mandible obtained by CT scanning. Angle Orthod 1998;68(6):557-562

34 Weijs WA, Hillen B. Relationships between masticatory muscle cross-section and skull shape. J Dent Res 1984;63(9):1154-1157

35 Satiroğlu F, Arun T, Işik F. Comparative data on facial morphology and muscle thickness using ultrasonography. Eur J Orthod 2005;27(6):562-567

36 Ringqvist M. Isometric bite force and its relation to dimensions of the facial skeleton. Acta Odontol Scand 1973;31(1):35-42

37 Ingervall B, Helkimo E. Masticatory muscle force and facial morphology in man. Arch Oral Biol 1978;23(3):203-206

38 Hannam AG, Wood WW. Relationships between the size and spatial morphology of human masseter and medial pterygoid muscles, the craniofacial skeleton, and jaw biomechanics. Am J Phys Anthropol 1989;80(4):429-445

39 Kiliaridis S, Kälebo P. Masseter muscle thickness measured by ultrasonography and its relation to facial morphology. J Dent Res 1991;70(9):1262-1265

40 Van Spronsen PH, Weijs WA, Prahl-Andersen B, Valk J, Van Finkel F. Relationships between jaw muscle cross-sections and normal craniofacial morphology, studied with magnetic resonance imaging. Eur J Orthod 1991;13:351-361

41 Bakke M, Tuxen A, Vilmann P, Jensen BR, Vilmann A, Toft M. Ultrasound image of human masseter muscle related to bite force, electromyography, facial morphology, and occlusal factors. Scand J Dent Res 1992;100(3):164-171

42 Kiliaridis S. Masticatory muscle influence on craniofacial growth. Acta Odontol Scand 1995;53(3):196-202

43 Forster CM, Sunga E, Chung $\mathrm{CH}$. Relationship between dental arch width and vertical facial morphology in untreated adults. Eur J Orthod 2008;30(3):288-294

44 van Spronsen PH, Weijs WA, Valk J, Prahl-Andersen B, van Ginkel FC. A comparison of jaw muscle cross-sections of longface and normal adults. J Dent Res 1992;71(6):1279-1285

45 Weijs WA, Hillen B. Correlations between the cross-sectional area of the jaw muscles and craniofacial size and shape. Am J Phys Anthropol 1986;70(4):423-431

46 Benington PC, Gardener JE, Hunt NP. Masseter muscle volume measured using ultrasonography and its relationship with facial morphology. Eur J Orthod 1999;21(6):659-670

47 Schulhof RJ, Lestrel PE, Walters R, Schuler R. The mandibular dental arch: part III. Buccal expansion. Angle Orthod 1978;48(4):303-310 